\title{
Mycobacterium avium complex lung disease in a patient treated with an immune checkpoint inhibitor: A case report
}

\author{
YUSUKE YAMABA $^{1}$, OSAMU TAKAKUWA ${ }^{1}$, YUSAKU TOMITA ${ }^{2}$, SOTA OWAKI ${ }^{1}$, \\ KAZUKI YAMADA $^{1}$, EIJI KUNII ${ }^{1}$, YUTAKA ITO ${ }^{3}$, KYOJI SENOO ${ }^{2}$ and KENJI AKITA ${ }^{1}$ \\ Departments of ${ }^{1}$ Respiratory Medicine and ${ }^{2}$ Gastroenterology, Nagoya City University West Medical Center, \\ Nagoya, Aichi 462-8508; ${ }^{3}$ Department of Respiratory Medicine, Allergy and Clinical Immunology, \\ Nagoya City University Graduate School of Medical Sciences, Nagoya, Aichi 464-0083, Japan
}

Received August 29, 2021; Accepted October 18, 2021

DOI: $10.3892 / \mathrm{mco} .2021 .2470$

\begin{abstract}
Immune checkpoint inhibitors (ICIs) are becoming widely used for the treatment of various types of cancer. However, characteristic side effects, which are referred to as immune-related adverse events, may appear, and they have important clinical implications for the management of patients treated with ICIs. The development of mycobacterial infections has also been reported, but they have mostly been seen in cases with tuberculosis, and only a few cases involved non-tuberculous mycobacteriosis. We herein present the case of an 82-year-old man who was treated with nivolumab for gastric cancer. After the 22nd course of the treatment, the patient experienced loss of appetite for 1 week, and infiltration shadows were observed in the lower lobe of the left lung. Treatment for bacterial pneumonia was ineffective, and the lung field shadow gradually worsened. Mycobacterium intracellulare was detected in two consecutive sputum cultures. Thus, the patient was diagnosed with Mycobacterium avium complex (MAC) lung disease, and treatment for MAC infection was thus initiated, with subsequent improvement of the patient's condition and infiltration shadows. At 7 months after the start of treatment, the sputum cultures became negative for acid-fast bacilli. Since MAC lung disease may develop acutely during immunotherapy with ICIs, clinicians should include it in the differential diagnoses for pneumonia during immunotherapy with ICIs.
\end{abstract}

\section{Introduction}

Immune checkpoints constitute one of the most important mechanisms for regulating the activation of the immune

Correspondence to: Dr Osamu Takakuwa, Department of Respiratory Medicine, Nagoya City University West Medical Center, 1-1-1 Hirate-cho, Kita-ku, Nagoya, Aichi 462-8508, Japan

E-mail: takakuwa@med.nagoya-cu.ac.jp

Key words: non-tuberculous mycobacteria, Mycobacterium avium complex, immune checkpoint inhibitors, nivolumab, pneumonia system (1). In cancer, the overexpression of programmed cell death-ligand 1 (PD-L1) on cancer cells and/or in the cancer microenvironment leads to the neutralization of activated programmed cell death-1 (PD-1)-positive cancer-specific T cells (2). Therapies with antibodies targeting PD-1 and its ligands restore T-cell functions. The effectiveness of these therapies has been widely demonstrated in clinical trials, and immune checkpoint inhibitors (ICIs) are becoming widely used for the treatment of various cancers (3). However, characteristic side effects, which are referred to as immune-related adverse events, may appear (4-6), and they have important clinical implications for the management of patients treated with ICIs. Furthermore, complications from infections have recently been reported to be an important issue in patients receiving treatment with ICIs (7). The development of mycobacterial infections has also been reported, but they have mostly been seen in cases with tuberculosis (1,8-10), and only a few cases involved non-tuberculous mycobacteriosis (11).

We herein report a case of Mycobacterium avium complex lung disease (MAC-LD) that developed rapidly in a patient who was being treated with nivolumab for gastric cancer.

\section{Case report}

An 82-year-old man visited the Nagoya City University West Medical Center (Nagoya, Japan) in March 2017 with a chief complaint of epigastric pain that started 3 months earlier, and he was diagnosed with stage IV gastric cancer. Tegafur/gimeracil/oteracil (TS-1) was administered as first-line chemotherapy (40 mg twice/day, consisting of 42-day cycles of 28-day consecutive administration followed by 14-day drug holiday, for a total of 9 cycles), and paclitaxel was administered as the second-line chemotherapy $\left(65 \mathrm{mg} / \mathrm{m}^{2}\right.$ on days 1,8 and 15 every 4 weeks for a total of 2 cycles), but neither treatment was effective. Due to increased bleeding from the tumor, the patient received radiation therapy (45 Gy) to control the bleeding. In October 2018, the patient was initiated on nivolumab (240 $\mathrm{mg}$ every 4 weeks) as the third-line chemotherapy; the nivolumab treatment induced a partial response, and was thus continued. After the 22nd course of the treatment, the patient experienced loss of appetite for 1 week, and was then referred to the Department of Respiratory Medicine of the Nagoya 
City University West Medical Center due to the appearance of infiltration shadows in the lower lobe of the left lung on chest X-ray (Fig. 1A) and chest CT (Fig. 2A) images. On the chest CT images taken 3 months ago, no abnormality was found bilaterally in the lung fields. The patient had no fever, and the blood test results revealed a white blood cell count of 5,200/ $\mu \mathrm{l}$ (normal range, 3,300-8,600/ $\mu \mathrm{l}$ ) and a C-reactive protein level of $0.6 \mathrm{mg} / \mathrm{dl}$ (normal range, $0.0-0.3 \mathrm{mg} / \mathrm{dl}$ ). The patient received moxifloxacin per os $400 \mathrm{mg} /$ day for 7 days on suspicion of bacterial pneumonia, but no treatment was administered subsequently as his condition and inflammatory response on blood tests were stable. One month later, the CT findings revealed an exacerbation, the infiltrative shadow in the lower left lobe had expanded, and other inflammatory shadows appeared in the left lingula and the lower lobe of the right lung (Fig. 2B); however, no fever or oxygen desaturation were observed. The results of the blood examination that was performed at that time are listed in Table I. No obvious abnormalities were observed, except for a slight increase in the C-reactive protein level to $3.2 \mathrm{mg} / \mathrm{dl}$. Mycobacterium intracellulare was detected twice in sputum cultures performed at the time, and no other causative bacteria were detected. The shadows were found mainly in the lower lobe of the left lung, and not in the middle lobe, lingula or lung apex, where lesions are usual observed in MAC-LD, so treatment for bacterial pneumonia was again administered. Meropenem at a dose of $1,000 \mathrm{mg} /$ day was selected as an antibiotic and the nivolumab treatment was discontinued; however, the lung shadows were further exacerbated after 4 weeks (Fig. 2C) and the case was diagnosed as MAC-LD. The standard treatment for MAC-LD is clarithromycin $500 \mathrm{mg}$ twice per day, rifampicin $10 \mathrm{mg} / \mathrm{kg}$ per day and ethambutol $15 \mathrm{mg} / \mathrm{kg}$ per day (12). Since the patient weighed $45 \mathrm{~kg}$, the standard doses for rifampicin and ethambutol were 450 and $675 \mathrm{mg} /$ day, respectively. However, as the patient was at an advanced age and suffered from gastric cancer, a decision was made to start with a dose lower than the standard dose of each drug, and treatment with all three drugs was initiated: Clarithromycin $600 \mathrm{mg} /$ day, rifampicin $300 \mathrm{mg} /$ day and ethambutol $500 \mathrm{mg} /$ day. Since it became difficult to continue treatment due to severe nausea as a side effect of the treatment, the medication schedule was changed from daily to 3 times per week, after which time the nausea markedly improved and the patient was able to continue receiving treatment. At 3 months after the start of medication, the lung shadows improved (Fig. 2D), and at 7 months after the start of treatment the sputum cultures became negative for acid-fast bacilli. Although resumption of nivolumab was considered, the patient declined treatment. He is currently being followed up and is on vonoprazan fumarate ( $20 \mathrm{mg}$ /day), but receives no chemotherapy for gastric cancer. An abdominal CT examination in August 2021 revealed thickening of the gastric wall, raising the suspicion of disease recurrence. The date of the last follow-up visit was 19 October 2021, at which time no infiltrative shadow in the lung was detected.

\section{Discussion}

We herein resent a case of MAC-LD that occurred during ICI immunotherapy. In a previous report, the underlying disease of the patients who developed MAC-LD during ICI
Table I. Laboratory data on admission.

\begin{tabular}{|c|c|}
\hline Laboratory parameters & Values (normal range) \\
\hline \multicolumn{2}{|l|}{ Hematology } \\
\hline White blood cell count, $/ \mu 1$ & $5,040(3,300-8,600)$ \\
\hline Neutrophils, $\%$ & $72.8(37-73)$ \\
\hline Lymphocytes, \% & $19.2(20-55)$ \\
\hline Monocytes, \% & $5.0(3-10)$ \\
\hline Eosinophils, \% & $2.8(0-11)$ \\
\hline Basophils, \% & $0.2(0-2)$ \\
\hline Red blood cell count, $\mathrm{x} 10^{4} / \mu \mathrm{l}$ & $321(435-555)$ \\
\hline Hemoglobin, g/dl & $11.2(13.7-16.8)$ \\
\hline Platelet count, $\mathrm{x} 10^{4} / \mu 1$ & $22.5(15.8-34.8)$ \\
\hline \multicolumn{2}{|l|}{ Biochemistry } \\
\hline Total protein, g/dl & $7.3(6.6-8.1)$ \\
\hline Albumin, g/dl & $3.1(4.1-5.1)$ \\
\hline Total bilirubin, mg/dl & $0.4(0.4-1.5)$ \\
\hline Alkaline phosphatase, IU/l & $447(106-322)$ \\
\hline Aspartate amino transferase, IU/l & $38(13-30)$ \\
\hline Alanine aminotransferase, IU/1 & $25(10-42)$ \\
\hline Lactate dehydrogenase, IU/1 & $147(124-222)$ \\
\hline Creatinine kinase, IU/1 & $50(59-248)$ \\
\hline Blood urine nitrogen, mg/dl & $14.3(8-20)$ \\
\hline Creatinine, $\mathrm{mg} / \mathrm{dl}$ & $0.79(0.65-1.07)$ \\
\hline $\mathrm{Na}, \mathrm{mEq} / \mathrm{l}$ & $134(138-145)$ \\
\hline $\mathrm{K}, \mathrm{mEq} / \mathrm{l}$ & $3.7(3.6-4.8)$ \\
\hline $\mathrm{Cl}, \mathrm{mEq} / \mathrm{l}$ & $101(101-108)$ \\
\hline $\mathrm{Ca}, \mathrm{mg} / \mathrm{dl}$ & $8.2(8.8-10.1)$ \\
\hline Glucose, mg/dl & $135(73-109)$ \\
\hline \multicolumn{2}{|l|}{ Serology } \\
\hline C-reactive protein, $\mathrm{mg} / \mathrm{dl}$ & $3.2(0.0-0.3)$ \\
\hline \multicolumn{2}{|l|}{ Tumor markers } \\
\hline Carcinoembryonic antigen, $\mathrm{ng} / \mathrm{ml}$ & $1.9(0.0-5.0)$ \\
\hline Carbohydrate antigen $19-9, \mathrm{U} / \mathrm{ml}$ & $11.0(0.0-37.0)$ \\
\hline
\end{tabular}

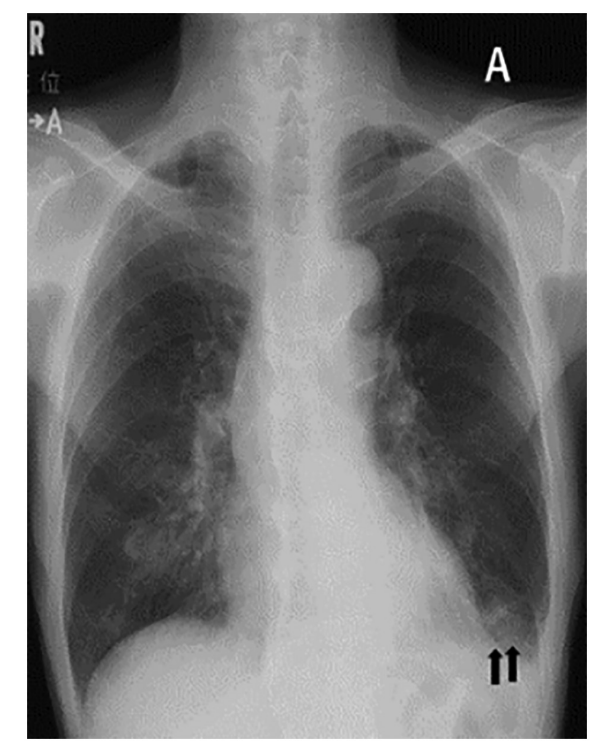

Figure 1. Chest X-ray showing infiltration shadows in the left lower lung field (arrows) (A). 

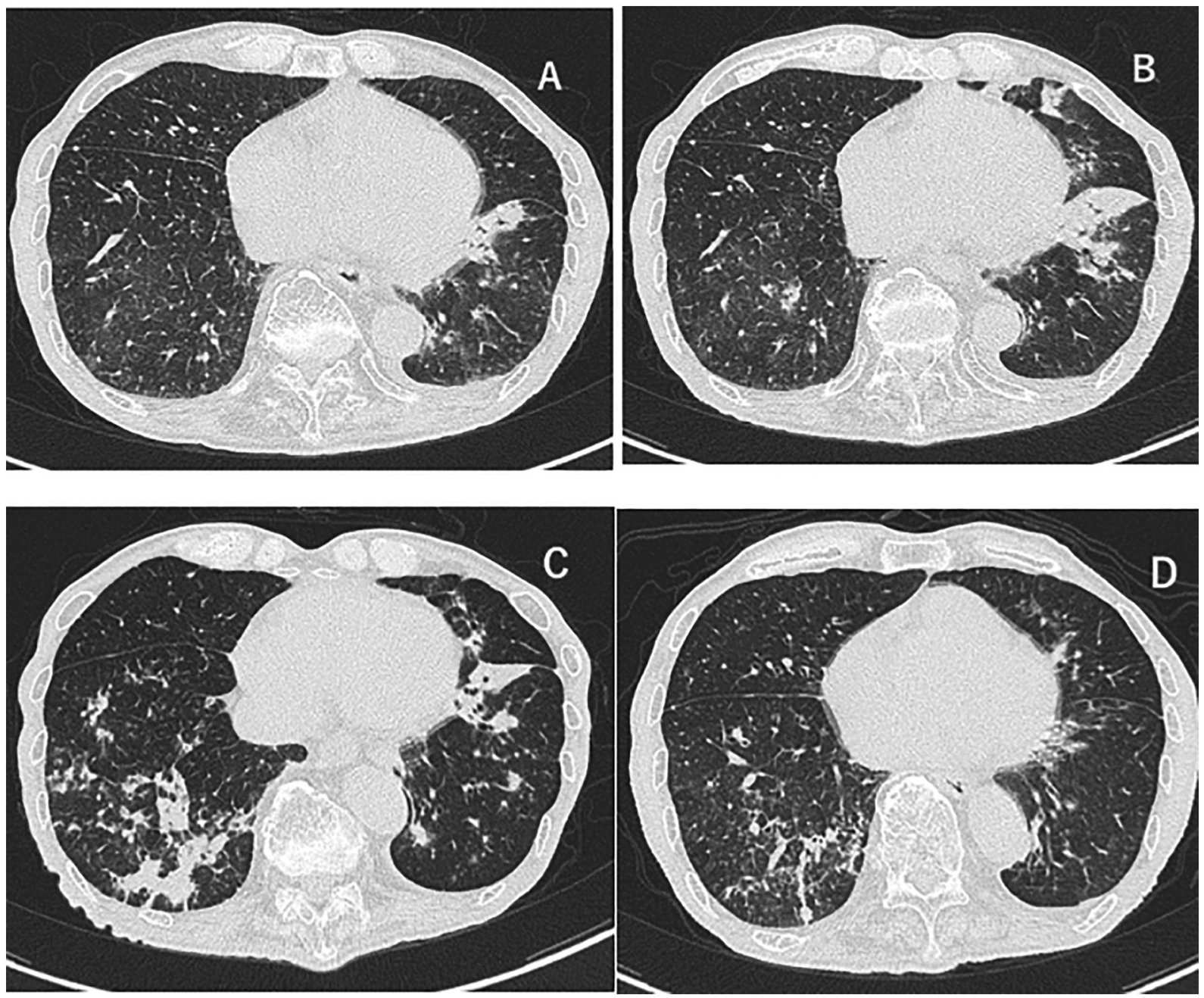

Figure 2. Chest CT images. CT examination was performed (A) during the patient's first visit to the Department of Respiratory Medicine, (B) 1 month later, (C) 2 months later, before the start of MAC treatment and (D) 3 months after the start of MAC treatment. The infiltration shadows in the lower left lobe, which worsened before MAC treatment (A-C), significantly improved after the start of the treatment (D). MAC, Mycobacterium avium complex.

immunotherapy was lung cancer (11). In the present case, the underlying malignant disease was gastric cancer, and no underlying lung disease was found. This indicated that MAC-LD may develop during ICI immunotherapy for various types of cancer, and is not limited to only lung cancer.

In this case, the clinical course was more acute than the usual course experienced in MAC-LD (13). The exact mechanism of MAC-LD is unknown, but anti-PD-1/PD-L1 antibodies are known to have potential antibacterial effects mediated by the upregulation of T-cell-mediated immunity (14). In fact, there is a case report in which the coexisting Mycobacterium abscessus lung disease improved when nivolumab was used to treat lung cancer (15). On the other hand, some cases of rapid-onset Mycobacterium tuberculosis (MTB) infection occurring during ICI immunotherapy have been reported $(1,8-10)$. In MTB, inhibition of the PD-1/PD-L1 pathway causes the upregulation of interferon-g production (16). While this mechanism could be expected to suppress MTB, it may also potentially lead to a hypersensitivity reaction to microorganisms that is similar to immune reconstitution inflammatory syndrome due to the inhibition of the PD-1/PD-L1 pathway (8). Indeed, transbronchial pulmonary biopsy specimens from patients who developed MTB during ICI immunotherapy have been reported to exhibit diffuse lymphocyte infiltration within the specimens (8). Another hypothesis attributes MTB infection to immune checkpoint-related lymphopenia (9); however, our patient did not develop lymphopenia during the clinical course. Therefore, it was inferred that MAC-LD may have developed rapidly due to a hypersensitivity reaction. In addition, the time period from the start of ICI treatment to the onset of MAC-LD was 20 months. In three previously reported cases (11), this time period was also between 17 and 19 months. This indicates that attention should be paid to long-term MAC-LD complications during ICI immunotherapy. Interestingly, all three previously reported cases (11) had a history of radiation therapy. In the present case, part of the lower left lobe was included in the radiation field of the radiation therapy for gastric cancer. It is unclear to what extent a medical history of radiation is implicated in the development of MAC-LD during ICI treatment, and further case reports are needed to compare findings and outcomes.

In patients with non-cavitary nodular/bronchiectatic macrolide-susceptible MAC-LD, a thrice-weekly macrolide-based regimen is recommended rather than a daily macrolide-based regimen (12). In the present case, daily 
therapy was first selected due to the acute onset during gastric cancer treatment. Owing to severe treatment-related nausea, the patient was switched to a thrice-weekly regimen, without compromised effectiveness. MAC treatment may be difficult to continue due to various factors, such as old age and the presence of cancer or other comorbidities. Intermittent therapy may also be effective, even in cases such as the present, in which MAC-LD occurred during ICI immunotherapy.

In conclusion, we herein present a case of MAC-LD that occurred in a patient with gastric cancer receiving ICI immunotherapy. It is important for clinicians to include MAC-LD as one of the differential diseases for pneumonia during ICI immunotherapy.

\section{Acknowledgements}

Not applicable.

\section{Funding}

No funding was received.

\section{Availability of data and materials}

The datasets used and/or analyzed during the current study are available from the corresponding author on reasonable request.

\section{Authors' contributions}

YY was involved in manuscript writing and acquisition of data. OT wrote the manuscript. YT and KS treated the patient for gastric cancer. SO, KY, EK and YI were involved in the treatment of the patient and interpreted the CT imaging and the laboratory test results. YY, OT and KA confirm the authenticity of all the raw data. All the authors have read and approved the final manuscript.

\section{Ethics approval and consent to participate}

Not applicable.

\section{Patient consent for publication}

Written informed consent was obtained from the patient for the publication of the clinical data and any associated images.

\section{Competing interests}

The authors declare that they have no competing interests.

\section{References}

1. Picchi H, Mateus C, Chouaid C, Besse B, Marabelle A, Michot JM, Champiat S, Voisin AL and Lambotte O: Infectious complications associated with the use of immune checkpoint inhibitors in oncology: Reactivation of tuberculosis after anti PD-1 treatment. Clin Microbiol Infect 24: 216-218, 2018.

2. Boussiotis VA: Molecular and biochemical aspects of the PD-1 checkpoint pathway. N Engl J Med 375: 1767-1778, 2016.

3. Sunshine J and Taube JM: PD-1/PD-L1 inhibitors. Curr Opin Pharmacol 23: 32-38, 2015.

4. Brahmer J, Reckamp KL, Baas P, Crinò L, Eberhardt WE Poddubskaya E, Antonia S, Pluzanski A, Vokes EE, Holgado E, et al: Nivolumab versus docetaxel in advanced squamous-cell non-small-cell lung cancer. N Engl J Med 373: 123-135, 2015.

5. Borghaei H, Paz-Ares L, Horn L, Spigel DR, Steins M, Ready NE, Chow LQ, Vokes EE, Felip E, Holgado E, et al: Nivolumab versus docetaxel in advanced nonsquamous non-small-cell lung cancer. N Engl J Med 373: 1627-1639, 2015.

6. Michot JM, Bigenwald C, Champiat S, Collins M, Carbonnel F, Postel-Vinay S, Berdelou A, Varga A, Bahleda R, Hollebecque A, et al: Immune-related adverse events with immune checkpoint blockade: A comprehensive review. Eur J Cancer 54: 139-148, 2016.

7. Fujita K, Kim YH, Kanai O, Yoshida H, Mio T and Hirai T: Emerging concerns of infectious diseases in lung cancer patients receiving immune checkpoint inhibitor therapy. Respir Med 146: 66-70, 2019.

8. Fujita K, Terashima T and Mio T: Anti-PD1 antibody treatment and the development of acute pulmonary tuberculosis. J Thorac Oncol 11: 2238-2240, 2016.

9. Chu YC, Fang KC, Chen HC, Yeh YC, Tseng CE, Chou TY and Lai CL: Pericardial tamponade caused by a hypersensitivity response to tuberculosis reactivation after anti-PD-1 treatment in a patient with advanced pulmonary adenocarcinoma. $\mathrm{J}$ Thorac Oncol 12: e111-e114, 2017.

10. Takata S, Koh G, Han Y, Yoshida H, Shiroyama T, Takada H, Masuhiro K, Nasu S, Morita S, Tanaka A, et al: Paradoxical response in a patient with non-small cell lung cancer who received nivolumab followed by anti-Mycobacterium tuberculosis agents. J Infect Chemother 25: 54-58, 2019.

11. Fujita K, Yamamoto Y, Kanai O, Okamura M, Nakatani K and Mio T: Development of Mycobacterium avium complex lung disease in patients with lung cancer on immune checkpoint inhibitors. Open Forum Infect Dis 7: ofaa067, 2020.

12. Daley CL, Iaccarino JM, Lange C, Cambau E, Wallace RJ Jr, Andrejak C, Böttger EC, Brozek J, Griffith DE, Guglielmetti L, et al: Treatment of nontuberculous mycobacterial pulmonary disease: An official ATS/ERS/ESCMID/IDSA clinical practice guideline: Executive Summary. Clin Infect Dis 71: e1-e36, 2020.

13. Daley CL: Mycobacterium avium complex disease. Microbiol Spectr 5, 2017

14. Rao M, Valentini D, Dodoo E, Zumla A and Maeurer M: Anti-PD-1/PD-L1 therapy for infectious diseases: Learning from the cancer paradigm. Int J Infect Dis 56: 221-228, 2017.

15. Ishii S, Tamiya A, Taniguchi Y, Tanaka T, Abe Y, Isa SI, Tsuyuguchi K, Suzuki K and Atagi S: Improvement of Mycobacterium abscessus pulmonary disease after nivolumab administration in a patient with advanced non-small cell lung cancer. Intern Med 57: 3625-3629, 2018.

16. Jurado JO, Alvarez IB, Pasquinelli V, Martínez GJ, Quiroga MF, Abbate E, Musella RM, Chuluyan HE and García VE: Programmed death (PD)-1:PD-ligand 1/PD-ligand 2 pathway inhibits $\mathrm{T}$ cell effector functions during human tuberculosis. J Immunol 181: 116-125, 2008. 